PROCEEDINGS OF THE

AMERICAN MATHEMATICAL SOCIETY

Volume 133, Number 6, Pages 1617-1620

S 0002-9939(05)07720-8

Article electronically published on January 21, 2005

\title{
VECTOR BUNDLES ON A PRODUCT OF REAL ALGEBRAIC CURVES
}

\author{
J. BOCHNAK AND W. KUCHARZ
}

(Communicated by Michael Stillman)

\begin{abstract}
We study complex vector bundles on a product of nonsingular real algebraic curves.
\end{abstract}

Throughout this note the term real algebraic variety designates a locally ringed space isomorphic to an algebraic subset of $\mathbb{R}^{N}$, for some $N$, endowed with the Zariski topology and the sheaf of $\mathbb{R}$-valued regular functions. Recall that each Zariski locally closed subset of real projective space $\mathbb{P}^{N}(\mathbb{R})$ can be regarded as a real algebraic variety [3, Propositions 3.2.10, 3.4.3]. Morphisms of real algebraic varieties will be called regular maps. Every real algebraic variety carries also the Euclidean topology, which is determined by the usual metric topology on $\mathbb{R}$. Unless explicitly stated otherwise, all topological notions related to real algebraic varieties will refer to the Euclidean topology.

Given a real algebraic variety $X$, an interesting problem is the one of comparison between algebraic and topological vector bundles on $X$. We deal here with $\mathbb{C}$ vector bundles, identifying $\mathbb{C}$ with $\mathbb{R}^{2}$ and viewing it as a real algebraic variety. A topological $\mathbb{C}$-vector bundle $\xi$ on $X$ is said to admit an algebraic structure if it is topologically isomorphic to an algebraic subbundle of the trivial vector bundle with total space $X \times \mathbb{C}^{p}$, for some $p$ (equivalently, if $\xi$ is topologically isomorphic to the $\mathbb{C}$-vector bundle corresponding, in the usual way [11, 12], to a finitely generated projective module over the ring of regular functions from $X$ into $\mathbb{C}$ ) [3, 4]. It is known that every topological $\mathbb{C}$-vector bundle on the unit sphere

$$
S^{n}=\left\{\left(x_{0}, \ldots, x_{n}\right) \in \mathbb{R}^{n+1} \mid x_{0}^{2}+\cdots+x_{n}^{2}=1\right\}
$$

or projective space $\mathbb{P}^{n}(\mathbb{R})$ admits an algebraic structure [9], [3, p. 326], and [4] Example 2.3(a)]. However, the behavior of $\mathbb{C}$-vector bundles on other "simple" real algebraic varieties can be drastically different. For example, a topological $\mathbb{C}$-vector bundle on the $k$-fold product $S^{1} \times \cdots \times S^{1}$ admits an algebraic structure if and only if it is stably trivial [3, Corollary 12.6.6, and p. 326]. More generally, for "generic" compact nonsingular real algebraic curves $C_{1}, \ldots, C_{k}$, a topological $\mathbb{C}$-vector bundle on $C_{1} \times \cdots \times C_{k}$ admits an algebraic structure if and only if it is stably trivial (cf. [5, 6, 7, for the precise meaning of the adjective "generic").

Received by the editors December 6, 2001 and, in revised form, February 26, 2004.

2000 Mathematics Subject Classification. Primary 14P25, 19 E99.

Both authors were partially supported by the Volkswagen Stiftung (Research in Pairs at Oberwolfach). 
We have the following:

Theorem. Let $M_{i}$ be a compact smooth submanifold (of class $\mathcal{C}^{\infty}$ and without boundary) of $\mathbb{P}^{n_{i}}(\mathbb{R})$, with $2 \operatorname{dim} M_{i}+1 \leq n_{i}$ for $1 \leq i \leq k$. Then for each $i$ there exists a smooth embedding $e_{i}: M_{i} \rightarrow \mathbb{P}^{n_{i}}(\mathbb{R})$, arbitrarily close in the $\mathcal{C}^{\infty}$ topology to the inclusion map $M_{i} \hookrightarrow \mathbb{P}^{n_{i}}(\mathbb{R})$, such that $X_{i}=e_{i}\left(M_{i}\right)$ is a nonsingular Zariski closed subvariety of $\mathbb{P}^{n_{i}}(\mathbb{R})$ and every topological $\mathbb{C}$-line bundle on $X_{1} \times \cdots \times X_{k}$ admits an algebraic structure; assuming $\operatorname{dim} M_{i}=1$ for $1 \leq i \leq k$, every topological $\mathbb{C}$-vector bundle on $X_{1} \times \cdots \times X_{k}$ admits an algebraic structure. Furthermore, if $n_{1}=\cdots=n_{k}$ and $M_{1}=\cdots=M_{k}$, then one can take $e_{1}=\cdots=e_{k}$ and hence $X_{1}=\cdots=X_{k}$.

Let us recall a result of $\mathrm{R}$. Benedetti and A. Tognoli [2, Theorem 4.2]. For any compact smooth submanifold (without boundary) $M$ of $\mathbb{P}^{n}(\mathbb{R})$, with $2 \operatorname{dim} M+1 \leq$ $n$, there exists a smooth embedding $e: M \hookrightarrow \mathbb{P}^{n}(\mathbb{R})$, arbitrarily close in the $\mathcal{C}^{\infty}$ topology to the inclusion map $M \hookrightarrow \mathbb{P}^{n}(\mathbb{R})$, such that $X=e(M)$ is a nonsingular Zariski closed subvariety of $\mathbb{P}^{n}(\mathbb{R})$ and every topological $\mathbb{C}$-vector bundle on $X$ admits an algebraic structure (strictly speaking, 2] deals with $\mathbb{R}$-vector bundles and with $\mathbb{R}^{n}$ instead of $\mathbb{P}^{n}(\mathbb{R})$, but a straightforward modification of the proof yields the assertion just stated). This does not directly imply our theorem.

In preparation for the proof we shall review a certain construction. Let $X$ be a nonsingular real algebraic variety. A nonsingular complexification of $X$ is a pair $(V, j)$, where $V$ is a nonsingular complex quasiprojective variety defined over $\mathbb{R}$ and $j: X \rightarrow V$ is an injective map such that the set $V(\mathbb{R})$ of real points of $V$ is Zariski dense in $V, j(X)=V(\mathbb{R})$, and $j$, viewed as a map from $X$ onto $V(\mathbb{R})$, is a regular isomorphism of real algebraic varieties. Define $H_{\mathrm{alg}}^{2}(V, \mathbb{Z})$ to be the subgroup of $H^{2}(V, \mathbb{Z})$ consisting of all cohomology classes of the form $c_{1}(L)$, where $L$ is a complex algebraic line bundle on $V$ and $c_{1}(-)$ stands for the first Chern class. Set

$$
H_{\mathbb{C}-\text { alg }}^{2}(X, \mathbb{Z})=j^{*}\left(H_{\text {alg }}^{2}(V, \mathbb{Z})\right),
$$

where $j^{*}: H^{*}(V, \mathbb{Z}) \rightarrow H^{*}(X, \mathbb{Z})$ is the homomorphism induced by $j$. One readily checks that $H_{\mathbb{C}-\text { alg }}^{2}(X, \mathbb{Z})$ does not depend on the choice of $(V, j)$ [4. Given a topological $\mathbb{C}$-line bundle $\lambda$ on $X$,

$$
\lambda \text { admits an algebraic structure } \Leftrightarrow c_{1}(\lambda) \in H_{\mathbb{C}-\text { alg }}^{2}(X, \mathbb{Z})
$$

4]. For any regular map $f: X \rightarrow Y$ between nonsingular real algebraic varieties, one has (cf. [4])

$$
f^{*}\left(H_{\mathbb{C}-\text { alg }}^{2}(Y, \mathbb{Z})\right) \subseteq H_{\mathbb{C}-\text { alg }}^{2}(X, \mathbb{Z}) .
$$

If $Y_{1}, \ldots, Y_{n}$ are connected nonsingular real algebraic varieties, then

$$
H^{2}\left(Y_{1} \times \cdots \times Y_{n}, \mathbb{Z}\right)=\sum_{i<j} p_{i j}^{*}\left(H^{2}\left(Y_{i} \times Y_{j}, \mathbb{Z}\right)\right),
$$

where $p_{i j}: Y_{1} \times \cdots \times Y_{n} \rightarrow Y_{i} \times Y_{j}$ is the canonical projection, and hence (2) implies

$$
H_{\mathbb{C}-\text { alg }}^{2}\left(Y_{1} \times \cdots \times Y_{n}, \mathbb{Z}\right)=H^{2}\left(Y_{1} \times \cdots \times Y_{n}, \mathbb{Z}\right),
$$

provided $H_{\mathbb{C}-\text { alg }}^{2}\left(Y_{i} \times Y_{j}, \mathbb{Z}\right)=H^{2}\left(Y_{i} \times Y_{j}, \mathbb{Z}\right)$ for all $i<j$.

Note that

$$
\sigma: \mathbb{P}^{n}(\mathbb{C}) \times \mathbb{P}^{n}(\mathbb{C}) \rightarrow \mathbb{P}^{n}(\mathbb{C}) \times \mathbb{P}^{n}(\mathbb{C}), \sigma(x, y)=(\bar{y}, \bar{x}),
$$


where the bar indicates complex conjugation, is an antiholomorphic involution. Thus $\mathbb{P}^{n}(\mathbb{C}) \times \mathbb{P}^{n}(\mathbb{C})$ can be regarded as a complex projective variety defined over $\mathbb{R}$, whose set of real points, denoted here by $\mathbb{P}^{n}(\mathbb{C})_{\mathbb{R}}$, coincides with the set of fixed points of the involution $\sigma$. Clearly, the map $\mathbb{P}^{n}(\mathbb{C}) \rightarrow \mathbb{P}^{n}(\mathbb{C})_{\mathbb{R}}, x \rightarrow \sigma(x, x)=(x, \bar{x})$, is a homeomorphism. Furthermore, by construction,

$$
H_{\mathbb{C}-\text { alg }}^{2}\left(\mathbb{P}^{n}(\mathbb{C})_{\mathbb{R}}, \mathbb{Z}\right)=H^{2}\left(\mathbb{P}^{n}(\mathbb{C})_{\mathbb{R}}, \mathbb{Z}\right) .
$$

Proof of the theorem. By [6, Corollary 1.6, Proposition 2.2], there exists a nonsingular algebraic curve $C$ in $\mathbb{P}^{2}(\mathbb{R})$ such that $C$ is connected and

$$
H_{\mathbb{C}-\text { alg }}^{2}(C \times C, \mathbb{Z})=H^{2}(C \times C, \mathbb{Z}) .
$$

Choose a positive integer $r$ with the property that the group $H^{q}\left(M_{i}, \mathbb{Z}\right)$ can be generated by $r$ elements for $1 \leq q \leq 2$ and $1 \leq i \leq k$. Let $\ell$ be a positive integer satisfying $\operatorname{dim} M_{i}+1 \leq \ell$ for $1 \leq i \leq k$. Setting

$$
B=\left(\mathbb{P}^{\ell}(\mathbb{C})_{\mathbb{R}}\right)^{r} \times C^{r},
$$

we can find a smooth map $\varphi_{i}: M_{i} \rightarrow B$ for which the induced homomorphism

$$
\varphi_{i}^{*}: H^{q}(B, \mathbb{Z}) \rightarrow H^{q}\left(M_{i}, \mathbb{Z}\right)
$$

is surjective for $1 \leq q \leq 2$ and $1 \leq i \leq k$ (see, for example, [8, Chap. V, 11.6, 11.9; Chap. VII, Sect. 12]).

Given a nonnegative integer $p$, we identify $\mathbb{P}^{n_{i}}(\mathbb{R})$ with the subset $\mathbb{P}^{n_{i}}(\mathbb{R}) \times\{0\}$ of $\mathbb{P}^{n_{i}}(\mathbb{R}) \times \mathbb{R}^{p} ;$ thus $\mathbb{P}^{n_{i}}(\mathbb{R}) \subseteq \mathbb{P}^{n_{i}}(\mathbb{R}) \times \mathbb{R}^{p}$. By [1, Theorem 2.8.4, Lemma 2.7.1], there exists a nonnegative integer $p$, a smooth embedding $\epsilon_{i}: M_{i} \rightarrow \mathbb{P}^{n_{i}}(\mathbb{R}) \times \mathbb{R}^{p}$, a nonsingular Zariski closed subvariety $Y_{i}$ of $\mathbb{P}^{n_{i}} \times \mathbb{R}^{p}$, and a regular map $\psi_{i}: Y_{i} \rightarrow B$ such that $Y_{i}=\epsilon_{i}\left(M_{i}\right), \epsilon_{i}$ is close in the $\mathcal{C}^{\infty}$ topology to the inclusion map $M_{i} \hookrightarrow$ $\mathbb{P}^{n_{i}}(\mathbb{R}) \times \mathbb{R}^{p}$, and $\varphi_{i}$ is homotopic to $\psi_{i} \circ \epsilon_{i}$ (the cited results of 1 are applicable since every homology class in $H_{j}(B, \mathbb{Z} / 2)$, for $0 \leq j \leq \operatorname{dim} B$, can be represented by a Zariski closed subvariety of $B$ ). In particular, the induced homomorphism

$$
\psi_{i}^{*}: H^{q}(B, \mathbb{Z}) \rightarrow H^{q}\left(Y_{i}, \mathbb{Z}\right)
$$

is surjective for $1 \leq q \leq 2$. Since $2 \operatorname{dim} Y_{i}+1 \leq n_{i}$, one can find an algebraic embedding $\eta_{i}: Y_{i} \rightarrow \mathbb{P}^{n_{i}}(\mathbb{R})$ (this means that $\eta_{i}\left(Y_{i}\right)$ is a Zariski closed subvariety of $\mathbb{P}^{n_{i}}(\mathbb{R})$ and $\eta_{i}: Y_{i} \rightarrow \eta_{i}\left(Y_{i}\right)$ is a regular isomorphism), close to $\pi_{i} \mid Y_{i}$, where $\pi_{i}: \mathbb{P}^{n_{i}}(\mathbb{R}) \times \mathbb{R}^{p} \rightarrow \mathbb{P}^{n_{i}}(\mathbb{R})$ is the canonical projection; cf. [10, pp. 21, 22]. By construction, $e_{i}=\eta_{i} \circ \epsilon_{i}: M_{i} \rightarrow \mathbb{P}^{n_{i}}(\mathbb{R})$ is a smooth embedding, close in the $\mathcal{C}^{\infty}$ topology to the inclusion map $M_{i} \hookrightarrow \mathbb{P}^{n_{i}}(\mathbb{R}), X_{i}=e_{i}\left(M_{i}\right)=\eta_{i}\left(Y_{i}\right)$ is a nonsingular Zariski closed subvariety of $\mathbb{P}^{n_{i}}(\mathbb{R})$, and $f_{i}=\psi_{i} \circ \eta_{i}^{-1}: X_{i} \rightarrow B$ is a regular map for which the induced homomorphism

$$
f_{i}^{*}: H^{q}(B, \mathbb{Z}) \rightarrow H^{q}\left(X_{i}, \mathbb{Z}\right)
$$

is surjective for $1 \leq q \leq 2$.

Consider the regular map

$$
f=f_{1} \times \cdots \times f_{k}: X_{1} \times \cdots \times X_{k} \rightarrow B^{k} .
$$

Clearly, the induced homomorphism

$$
f^{*}: H^{2}\left(B^{k}, \mathbb{Z}\right) \rightarrow H^{2}\left(X_{1} \times \cdots \times X_{k}, \mathbb{Z}\right)
$$

is surjective. Furthermore, it easily follows from (3), (4), (5) that

$$
H_{\mathbb{C}-\text { alg }}^{2}\left(B^{k}, \mathbb{Z}\right)=H^{2}\left(B^{k}, \mathbb{Z}\right) .
$$


Combining (2), (6), (7), we obtain

$$
H_{\mathbb{C}-\text { alg }}^{2}\left(X_{1} \times \cdots \times X_{k}, \mathbb{Z}\right)=H^{2}\left(X_{1} \times \cdots \times X_{k}, \mathbb{Z}\right)
$$

which in view of (1) implies that every topological $\mathbb{C}$-line bundle on $X_{1} \times \cdots \times X_{k}$ admits an algebraic structure. Thus the first part of the theorem is proved.

Clearly, assuming $n_{1}=\cdots=n_{k}$ and $M_{1}=\cdots=M_{k}$, we can take $e_{1}=\cdots=e_{k}$ and $X_{1}=\cdots=X_{k}$.

Suppose now $\operatorname{dim} M_{i}=1$ for $1 \leq i \leq k$. Without loss of generality, we may assume that each $M_{i}$ is connected. Then the real algebraic variety $X=X_{1} \times \cdots \times X_{k}$ is connected, $H^{*}(X, \mathbb{Z})$ has no torsion, and the ring $H^{\text {even }}(X, \mathbb{Z})=\bigoplus_{j \geq 0} H^{2 j}(X, \mathbb{Z})$ is generated by $H^{0}(X, \mathbb{Z})$ and $H^{2}(X, \mathbb{Z})$. Since, in addition to these facts, condition (8) holds, it follows from [5] Proposition 2.4] that every topological $\mathbb{C}$-vector bundle on $X$ admits an algebraic structure. This completes the proof.

\section{REFERENCES}

[1] S. Akbulut and H. King, Topology of Real Algebraic Sets, Math. Sci. Research Institute Publ. 25, New York Berlin Heidelberg, Springer, 1992. MR1225577 (94m:57001)

[2] R. Benedetti and A. Tognoli, On real algebraic vector bundles, Bull. Sci. Math. 104 (1980), 89-112. MR0560747 (81e:14009)

[3] J. Bochnak, M. Coste and M.-F. Roy, Real Algebraic Geometry, Ergebnisse der Mathematik und ihrer Grenzgebiete, Folge 3, Vol. 36, Berlin Heidelberg New York, Springer, 1998. MR 1659509(2000a:14067)

[4] J. Bochnak, M. Buchner and W. Kucharz, Vector bundles on real algebraic varieties, KTheory 3 (1989), 271-298, Erratum, K-Theory 4 (1990), p. 103. MR.1040403 (91b:14075)

[5] J. Bochnak and W. Kucharz, Vector bundles on a product of real cubic curves, K-Theory 6 (1992), 487-497. MR1204824 (94e:14077)

[6] J. Bochnak and W. Kucharz, Elliptic curves and real algebraic morphisms, J. Algebraic Geometry 2 (1993), 635-666. MR 1227471 (94e:14072)

[7] J. Bochnak, W. Kucharz, and R. Silhol, Morphisms, line bundles and moduli spaces in real algebraic geometry, Inst. Hautes Etudes Sci. Publ. Math. 86 (1997), 5-65. MR1608561 (99h:14055)

[8] G. E. Bredon, Geometry and Topology, New York Berlin Heidelberg, Springer, 1997. MR 1700700 (2000b:55001)

[9] R. Fossum, Vector bundles on spheres are algebraic, Invent. Math. 8 (1969), 222-225. MR 0250298 (40:3537)

[10] N. Ivanov, Approximation of smooth manifolds by real algebraic sets, Russian Math. Surveys 37 (1982), 1-59. MR0643764 (84i:57029)

[11] J.-P. Serre, Faisceaux algébriques cohérents, Ann. of Math. 61 (1955), 197-278. MR0068874 $(16: 953 \mathrm{c})$

[12] R. G. Swan, Vector bundles and projective modules, Trans. Amer. Math. Soc. 105 (1962), 264-277. MF $0143225(26: 785)$

Department of Mathematics, Vrije Universiteit, De Boelelaan 1081A, 1081 HV Amsterdam, The Netherlands

E-mail address: bochnak@cs.vu.nl

Department of Mathematics and Statistics, University of New Mexico, Albuquerque, New Mexico 87131-1141

E-mail address: kucharz@math.unm.edu 